Research Article

\title{
Effects of a Six-Week Progressive Resistance Training Program on Functional Fitness among Community-Dwelling Older Adults
}

Karla Caillouet *, Joshua Wludyga, Elgin Andrews

Exercise Science, Georgia Gwinnett College, 1000 University Center Lane, Lawrenceville, United States; E-Mails: kcaillouet@ggc.edu; jwludyga@ggc.edu; eandrews3@ggc.edu

* Correspondence: Karla A. Caillouet; E-Mail: kcaillouet@ggc.edu

Academic Editor: José M Cancela Carral

Special Issue: Physical Activity and Older Adults. Intervention Programs

\section{OBM Geriatrics}

2020, volume 4 , issue 4

doi:10.21926/obm.geriatr.2004138
Received: May 06, 2020

Accepted: November 05, 2020

Published: November 16, 2020

\begin{abstract}
Research has indicated that older adult participation in resistance training provides foundational strength for performance of activities of daily living. The purpose of this study was to investigate the effects of a six-week progressive resistance training intervention on functional fitness among community-dwelling older adults. From 2010-2015, twelve sessions of a six-week resistance training intervention included 95 community-dwelling older adult participants ( 37 male, 58 female; Mage=73.5+7.2). Resistance training interventions included bi-weekly, 1-hour sessions targeting all muscle groups. Assessments included arm curl, chair stand, back scratch, chair sit-n-reach, 2-minute step test, and 8 foot-up-and-go. The Wilcoxon Signed-Rank Test was performed to evaluate group differences between baseline and postintervention. Significant differences between pre- and post-assessments were found for upper- and lower-body strength $(z=6.81, p<.001 ; z=6.46, p<.001$, respectively); upper- and lower-body flexibility $(z=3.81, p<.001 ; z=4.85, p<.001$, respectively), aerobic endurance $(z=6.04, p<.001)$, and dynamic balance and agility $(z=5.52, p<.001)$. The resistance training intervention resulted in significant improvements in functional fitness among communitydwelling older adult participants. Abbreviations: 1-RM, 1 Repetition Maximum.
\end{abstract}

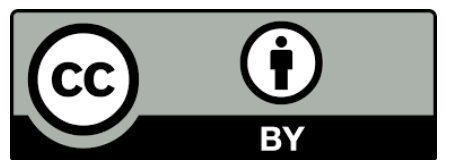

(C) 2020 by the author. This is an open access article distributed under the conditions of the Creative Commons by Attribution License, which permits unrestricted use, distribution, and reproduction in any medium or format, provided the original work is correctly cited. 


\section{Keywords}

Resistance training; aerobic endurance training; flexibility; dynamic balance and agility; functional fitness: older adults; aging

\section{Introduction}

Sarcopenia is characterized as a loss of skeletal muscle tissue and is often aging-related. Research suggests that the loss of muscle mass, strength, and function during aging can lead to increased risk for falls, decreased quality of life, increased risk for loss of independence, and increased risk of mortality [1, 2]. In addition, physiological deficits among the older adult population that have been associated with sarcopenia, such as muscle protein turnover and apoptosis, may contribute to increased healthcare costs [3]. When incorporated with additional exercise modalities including cardiovascular endurance training, flexibility training, and balance training, resistance training has been associated with improvements in symptoms associated with chronic conditions. The benefits of resistance training include, but are not limited to, improvements related to osteoarthritis, improved blood lipid profiles, improved glucose metabolism, improved bone health, reductions in blood pressure, improved body composition, and improved functional fitness [4-7].

Participation in a regular, progressive resistance training program can have a positive and profound impact on functional ability and independent living [8]. Functional fitness is related to the ability to perform activities associated with daily living such as walking, rising from a chair, lifting, reaching, climbing stairs, bending, and kneeling [4]. These daily functions require sufficient levels of fitness in strength, agility, endurance, and flexibility [9]. More specifically, activities such as walking, jogging, running, and stair climbing require muscular strength, endurance, balance, and agility. Standing up from a chair, bending, and kneeling all require muscular strength and flexibility [9]. Thus, the benefits of functional fitness include the ability to maintain independence and overall improved quality of life [9].

According to Burton and Sumukadas [10], functional independence is predicated by achieving proper maintenance of muscle function as a person ages. Participation in resistance training programs can provide safe and effective methods for building muscle and strength among the older adult population [11]. When compared to aerobic exercise, resistance training offers greater benefits for increased muscular quality, increased lean muscle mass, and increased muscular contractile units among older adults [12]. A position stand from the American College of Sports Medicine (ACSM) suggests older adults engage in resistance training a minimum of two days per week in order to maintain or improve musculoskeletal function [13]. Similarly, the newly formed position stand from the National Strength and Conditioning Association (NSCA) recommends older adults engage in regular strength training at 2-3 sets of 1-2 multi-joint exercises per major muscle group, achieving intensities of 70-85\% of 1 repetition maximum (1RM) [14].

Research has suggested that resistance training programs for older adults lasting 12 weeks or more have shown increases in muscle mass and strength [15-17]. However, Scanlon and colleagues [18] suggest that six weeks of resistance training among older adults is an acceptable period for demonstrating improvements in strength and muscle morphology. Despite these findings, minimal research has investigated the effects of short-term resistance training programs on older adults, 
with the intent on mitigating the risk of sarcopenia, improving functional fitness, and contributing to long-term physical independence. The purpose of this study was to investigate the effects of a six-week progressive resistance training program on functional fitness, including upper- and lowerbody strength, upper- and lower-body flexibility, aerobic endurance, and dynamic balance and agility among community-dwelling older adults.

\section{Materials and Methods}

\subsection{Methodology}

This study took place at a university in Northwest Florida as part of an aging and physical performance course in an undergraduate Exercise Science program. Approval from the Institutional Review Board was obtained prior to this study. Participants were recruited through word-of-mouth and brochure distribution to local senior centers and churches. All participants signed an Informed Consent. In addition to the Informed Consent, older adult participants completed a Health Activity Questionnaire [4] and obtained a Physician's Medical Clearance. From 2010 to 2015, the study was conducted each fall and spring semester, for a total of 12 semesters. Students assisting with the administration of the study intervention were junior-senior-level students pursuing a degree in Exercise Science. During the first seven weeks of any given semester, students participated in intensive instruction that included Senior Fitness Test administration protocol, proper exercise technique, and exercise prescription for special medical conditions among the older adult population. During the last seven weeks of any given semester, students partnered with a community-dwelling older adult volunteer to administer the six-week resistance training program. Students reviewed their older adult partner's Health Activity Questionnaire and the associated Physician's Medical Clearance to ascertain potential contraindications.

\subsection{Training Protocol}

All resistance training activities took place at the university's on-site fitness facilities. The program was supervised by the course instructor, who was an American Council on Exercise certified Personal Trainer, an Aerobics and Fitness Association of America certified Instructor, and a FallProof ${ }^{\mathrm{TM}}$ Balance and Mobility certified Instructor with more than eight years' experience working in the fitness industry. At least one graduate assistant completing graduate work in Exercise Physiology served as an assistant supervisor each semester. From 2010-2015, twelve separate sessions of a six-week progressive resistance program were implemented, resulting in a total of 95, generally healthy, ambulatory community-dwelling older adult participants ( 37 male, 58 female; Mage $=73.5 \pm 7.2$ ). Thus, there were twelve cohorts of participants throughout the six years the study was conducted. Mean body mass index for study participants was $29.67 \pm 5.26$ for males and $25.33 \pm 3.63$ for females. Mean height for male and female participants was $5^{\prime} 7 " \pm 3.18^{\prime \prime}$ and $5^{\prime} 3^{\prime \prime} \pm$ $2.9^{\prime \prime}$, respectively.

\subsection{Study Design}

Each resistance training program consisted of bi-weekly, one-hour sessions targeting major muscle groups for a total of 12 one-hour sessions in the six-week period. Sessions included a warm- 
up, a resistance training workout, and a cool down that incorporated low-intensity movements and static flexibility training. Intervention exercises included the leg press, leg extension, leg curl, lower back extension, hip abduction, hip adduction, compound row, latissimus dorsi pull-down, abdominal crunches, calf raises, triceps cable pushdowns, vertical chest press, bicep curls, lateral raises, front raises, and plantar and dorsi-flexion. However, exercises performed during the intervention may have been modified or omitted based upon individual older adult participant-reported contraindications. Each exercise included in the resistance training program was selected based upon its contribution to functional fitness. For example, adequate strength in hip abduction and adduction is indicated to positively impact lateral stability. Ankle strength gains earned through plantar- and dorsi-flexion are associated with improved balance and mobility. Chest strength is required for upper-body control during walking [4]. Knee extensor and calf strength are positively associated with gait speed, which is negatively associated with morbidity and mortality $[19,20]$.

Evidence indicates that healthy older adults may safely participate in training loads between 70 and $80 \%$ of $1-\mathrm{RM}$ in a resistance training exercise program [21]. Thus, resistance training loads for individual participants in this study were established using the $70-80 \%$ 1-RM estimation methodology from Baechle and Westcott [21]. This methodology requires that individual participants complete each exercise within 8-12 repetitions at a challenging weight. Challenging loads for each exercise were determined by participant feedback during completion of each exercise. All participants began the resistance training program at this level. To ensure utilization of the anaerobic energy system, repetitions were performed for six seconds each, focusing on two seconds for the concentric contraction, and four seconds for the eccentric contraction [21]. Student administrators timed each concentric and eccentric contraction and vocally counted repetitions during each exercise to encourage adherence to the program protocol.

Research suggests that resistance training programs should begin with larger muscle groups and progress to smaller muscle groups during a single training session [4, 21, 22]. In addition, single- and multi-joint exercises are recommended [22]. Thus, training administrators focused on multi-joint, large muscle groups at the beginning of each training session and ended training sessions with focus on single-joint, smaller muscle groups. Based upon recommendations from the American College of Sports Medicine [22], all participants were provided with one-to-two minutes of rest between each new exercise. Resistance training participants were also provided with a minimum of 30 seconds of rest between sets.

At the beginning of the intervention, participants completed one set of 8-15 repetitions. When a participant could complete twelve repetitions for the initial loads, another set was added. Resistance training loads were increased for each exercise when the participant could achieve two sets of 12 to 15 repetitions per exercise. These determinations were made according to participant performance and the feedback provided by the participant. Based on recommendations from Baechle and Westcott [21] for safe and efficient load progression, the progressive resistance training program included load increases of approximately $5 \%$. The load increase was accompanied by a decrease in repetitions and/or sets, depending upon participant tolerance. Once two sets of 12 to 15 repetitions of the increased load could be completed successfully, weight loads were again increased as tolerated and the cycle repeated. Resistance training exercises were performed using Nautilus Nitro ${ }^{\circledR}$ Plus strength training systems, a Precor ${ }^{\circledR}$ multi-gym 8-stack, ten station cable system for tricep pushdowns, dumbbells for bicep curls and shoulder exercises, and resistance bands for plantar and dorsiflexion exercises. 
The Senior Fitness Test is a functional fitness assessment designed for older adults 60 years of age and older. It is recognized for its validity and reliability and is accompanied by norm-referenced and criterion-referenced standards [9]. For the purpose of this study, assessments for upper- and lower-body strength, upper- and lower-body flexibility, aerobic endurance, and dynamic balance and agility were based upon functional fitness assessment protocols provided in the Senior Fitness Test Manual [9]. Trained Senior Fitness Test administrators assessed participants the week prior to the implementation of the resistance training program and again the week after the resistance training program was completed. Table 1 outlines the variables of interest associated with the Senior Fitness Test [9] and the related assessments.

\section{Outcome Measures}

A Wilcoxon Signed-Rank Test was performed to analyse group differences between baseline and post-intervention for upper- and lower-body strength, upper- and lower-body flexibility, aerobic endurance, and dynamic balance and agility $(p<0.05)$.

Table 1 Senior Fitness Test.

\begin{tabular}{ll}
\hline Variables of Interest & Assessment \\
\hline Upper-Body Strength & 30-Second Arm Curl \\
Lower-Body Strength & 30-Second Chair Stand \\
Aerobic Endurance & 2-Minute Step Test \\
Upper-Body Flexibility & Back Scratch \\
Lower-Body Flexibility & Chair Sit-and-Reach \\
Dynamic Balance and Agility & 8 Foot-Up-and-Go \\
\hline
\end{tabular}

\section{Results}

Ninety-five participants were recruited for the study, but some participants chose to omit some assessments due to contraindications. The difference in scores was approximately symmetrically distributed, as evaluated by a histogram with a superimposed normal curve. Significant differences between pre-and post-assessments were found for upper- and lower-body strength $(z=6.81, p$ $<.001 ; z=6.46, p<.001$, respectively), upper-and lower-body flexibility $(z=3.81, p<.001 ; z=4.85$, $p<.001$, respectively), aerobic endurance $(z=6.04, p<.001)$, and dynamic balance and agility $(z=$ $5.52, p<.001)$. A summary of each data analysis is provided below. Pre- and post-assessment median scores, along with differences, may be found in Table 2.

Table 2 Senior Fitness Test Pre- Post-Assessment Medians and Differences.

\begin{tabular}{lclll}
\hline Senior Fitness Test & Sample Size & $\begin{array}{l}\text { Pre- } \\
\text { Assessment }\end{array}$ & $\begin{array}{l}\text { Post- } \\
\text { Assessment }\end{array}$ & Difference \\
\hline 30-Second Arm Curl & 82 & 19 & 21 & $3^{* *}$ \\
30-Second Chair Stand & 78 & 14 & 17 & $2^{* *}$
\end{tabular}




\begin{tabular}{lcccc} 
Two-Minute Step Test & 78 & 96 & 108 & $11^{* *}$ \\
Back Scratch & 94 & -4.0 & -3.75 & $.50^{* *}$ \\
Chair Sit-and-Reach & 95 & 1.5 & 2.0 & $1.0^{* *}$ \\
8 Foot-Up-and-Go & 92 & 5.25 & 4.79 & $.44^{* *}$ \\
\hline
\end{tabular}

Notes: ${ }^{*} p<0.05 ;{ }^{* *} p<0.001$. Back scratch and sit-and-reach are reported in inches. Eight foot up-and-go is reported in seconds.

\subsection{0-Second Arm Curl}

There were 82 participants in the study that participated in the 30-second arm curl assessment. Of the 82 participants, 73 improved performance, 14 decreased performance, and eight experienced no improvement. There was a statistically significant increase in the number of repetitions performed $(M d n=3)$ from pre-assessment $(M d n=19)$ to post-assessment $(M d n=21), \mathrm{z}$ $=6.80, p<.001$.

\subsection{0-Second Chair Stand}

Ninety-two participants completed the pre- and post-assessments for the 30 -second chair stand. Of these, 72 improved performance, seven decreased performance, and 13 elicited no improvement. There was a statistically significant median increase in repetitions from pre-assessment $(M d n=14)$ to post-assessment $(M d n=17), \mathrm{z}=6.46, p<.001$.

\subsection{Two-Minute Step Test}

Of the 95 participants in the study, 78 were analyzed for the two-minute step test. Of the 78 participants in the two-minute step test, the intervention elicited improvement in 62 of the participants. Fourteen participants experienced a decrease in performance and two participants experienced no improvement. Data analysis determined that there was a statistically significant increase in the number of steps performed $(M d n=11)$ from pre-intervention $(M d n=96)$ when compared to post-intervention $(M d n=108)$. The resistance training intervention elicited a statistically significant median increase in two-minute step test performance, $z=6.04, p<.001$.

\subsection{Back Scratch}

Ninety-four participants in the study completed pre- and post-assessments for the back scratch. Of the 94, 58 participants improved upper body flexibility, 21 decreased upper body flexibility, and 15 participants showed no improvement. There was a statistically significant increase in upper body flexibility from pre-assessments ( $M d n=-4.0$ inches) to post-assessments ( $M d n=-3.75$ inches), $\mathrm{z}=$ $3.18, p<.001$.

\subsection{Chair Sit-and-Reach}


All participants in the study performed pre- and post-assessments for the chair sit-and-reach. Of the 95, 68 participants improved lower-body flexibility, 19 decreased lower-body flexibility, and eight demonstrated no change. Data analysis indicated a statistically significant increase in lowerbody flexibility from pre-assessments ( $M d n=1.5$ inches) when compared to post-assessments ( $M d n$ $=2.0), z=4.85, p<.001$.

\subsection{Foot Up-and-Go}

Ninety-two participants completed pre- and post-assessments for the 8 foot up-and-go. Of the 92 participants, 73 improved their time to complete the test, 17 increased their time to complete the test, and two indicated no improvement. There was a statistically significant decrease in time to perform the test from pre-assessments ( $M d n=5.25$ seconds) when compared to post-assessments $(M d n=4.79), z=-5.517, p<.001$.

\section{Discussion}

Participation in a regular resistance training program has demonstrated increased muscular strength, increased muscular endurance, improved functional abilities, improved health, and overall improved quality of life among older adults [23]. For previous studies lasting more than eight weeks, resistance training promotes improvements to both overall strength and lean muscle mass in older adult participants $[17,24]$. The findings in this study suggest that participation in a bi-weekly, progressive resistance training program may result in significant improvements in functional fitness in as little as six weeks. These results align with recommendations and expected benefits set forth by both ACSM and NSCA $[13,14]$. Interestingly, aerobic endurance also improved after participants completed the resistance training program. Typically, resistance training is not promoted as a means to improve aerobic endurance capacity. However, previous studies have noted resistance training can improve walking endurance and overall physical capacity to carry about aerobic endurancerelated tasks [25]. Regular resistance training also may enhance mitochondrial density and oxidative capacity of muscle tissue [26].

There are potentially numerous factors that contributed to improvements in muscular performance within the participants of this study. Sarcopenic factors such as pro-inflammatory cytokines, protein catabolism, and catabolic hormone release may all be positively influenced with resistance exercise [3, 14]. However, improvements to muscular performance in short-term resistance training interventions are often the result of a combination of neural adaptations rather than structural changes to the muscle [27]. Neuromuscular adaptations may include improved neural firing rates and greater synchronization of the timing of neural discharge during anaerobic activities [27]. Despite initial changes possibly limited to neuromuscular adaptations, the benefit of such changes could positively impact the quality of life of an older adult. Physical disability is a major disease state affecting numerous members of the older adult population, as the result of muscle weakness, frailty, and fall risk [13]. Based on the results of this study, short-term resistance exercise may mitigate the risk of falling within the population, regardless of body composition alterations within the first six weeks. More research is needed to fully understand the relationship between resistance exercise and its impact on performance in activities of daily living [14].

Flexibility benefits were also observed among the participants of this study. While daily stretching protocols may promote the greatest benefit to long-term maintenance of range of 
motion, regular resistance training is thought to have nearly equal benefit to improving flexibility in untrained individuals [28]. Promotion of moving a synovial joint through its full range of motion offers greater capacity for an individual to adequately move in any daily physical activity, whether that range of motion is accomplished via regular stretching exercises, resistance training, or a combination of both.

The significant improvements in functional fitness during the shorter six week time frame is noteworthy for participant adherence to the training program, since adherence is a key factor in impacting treatment effectiveness [29]. Previous research indicates continual benefits if participants were to maintain a resistance training program at or beyond a six-week duration $[2,11]$. Furthermore, this study adds to the accumulating evidence that community-dwelling older adults should engage in regular resistance training. In addition to the specific measures indicated in this study, older adults may also exhibit a reduction in fall risk, resulting from exercises targeting improvements in proprioception, strength, and dynamic balance [30, 31]. Research also indicates a significant association between exercise capacity and depression in this population [32]. The present study demonstrates significant improvements in exercise capacity, from both anaerobic and aerobic perspectives.

\section{Conclusions}

The significant outcomes from this study indicate that a six-week progressive resistance training program that targets all muscle groups may result in improved strength, flexibility, aerobic endurance, and dynamic balance and agility among community-dwelling older adult participants. While previous studies have suggested that more time in exercise program participation is needed to observe significant changes in functional fitness, outcomes from this study suggest that a sixweek progressive resistance training program may be sufficient to affect positive functional fitness change among community-dwelling older adults. These results may be promising to the older adult population, who may be motivated to participate in resistance training programs because positive results may occur within a short period of time. Future research should utilize similar progressive resistance training methodologies for older adults but should incorporate larger sample sizes and a control group.

\section{Acknowledgments}

We thank everyone who participated in this study.

\section{Author Contributions}

Karla Caillouet conceived, designed and carried out the data analysis and interpretation. Karla Caillouet wrote the first draft. Joshua Wludyga and Elgin Andrews critically discussed all the versions of the manuscript, contributed to manuscript content, and appraised the final version prior to submission.

\section{Competing Interests}

The authors declare no conflicts of interest. 


\section{References}

1. Montero-Fernandez N, Serra-Rexach JA. Role of exercise on sarcopenia in the elderly. Eur J Phys Rehabil Med. 2013; 49: 131-143.

2. Rosado MD, Tomas MT, Correia SC, Goncalves CR, de Abreu MH, Cardoso SF. Resistance training for muscle strength and lean mass in adults older than 60 Years: A systematic review. Indian J Med Res Pharm Sci. 2016; 3: 16-27.

3. Buford TW, Anton SD, Judge AR, Marzetti E, Wohlgemuth SE, Carter CS, et al. Models of accelerated sarcopenia: Critical pieces for solving the puzzle of age-related muscle atrophy. Ageing Res Rev. 2010; 9: 369-383.

4. Rose DJ. Physical activity instruction of older adults. 2nd ed. Champaign: Human Kinetics; 2011.

5. Ratamess NA, Alvar BA, Evetoch TE, Housh TJ, Ben Kibler W, Kraemer WJ, et al. Progression models in resistance training for healthy adults. Med Sci Sports Exerc. 2009; 41: 687-708.

6. Pescatello LS, Franklin BA, Fagard R, Farquhar WB, Kelley GA, Ray CA. Exercise and hypertension. Med Sci Sports Exerc. 2004; 36: 533-553.

7. Kohrt WM, Bloomfield SA, Little KD, Nelson ME, Yingling VR. Physical activity and bone health. Med Sci Sports Exerc. 2004; 36: 1985-1996.

8. Billson JH, Cilliers JF, Peiterse JJ, Shaw BS, Shaw I, Toriola AL. Comparison of home-and gymnasium-based resistance training on flexibility in the elderly. S African J Res Sport Phys Educ. 2011; 33: 1-9.

9. Rikli RE, Jones CJ. Senior Fitness Test Manual. 2nd ed. Champaign: Human Kinetics; 2013.

10. Burton LA, Sumaukadas D. Optimal management of sarcopenia. Clin Interv Aging. 2010; 5: 217228.

11. Peterson MD, Rhea MR, Sen A, Gordon PM. Resistance exercise for muscular strength in older adults: A meta-analysis. Ageing Res Rev. 2010; 9: 226-237.

12. Aagaard P, Suetta C, Caserotti P, Magnusson SP, Kjær M. Role of the nervous system in sarcopenia and muscle atrophy with aging: Strength training as a countermeasure. Scand J Med Sci Sports. 2010; 20: 49-64.

13. Chodzko-Zajko WJ, Proctor DN, Singh MA, Minson CT, Nigg CR, Salem GJ, et al. Exercise and physical activity for older adults. Med Sci Sports Exerc. 2009; 41: 1510-1530.

14. Fragala MS, Cadore EL, Dorgo S, Izquierdo M, Kraemer WJ, Peterson MD, et al. Resistance training for older adults: Position statement from the National Strength and Conditioning Association. J Strength Cond Res. 2019; 33: 2019-2052.

15. Bickel CS, Cross JM, Bamman MM. Exercise dosing to retain resistance training adaptations in young and older adults. Med Sci Sports Exerc. 2011; 43: 1177-1187.

16. Mueller M, Breil FA, Vogt M, Steiner R, Lippuner K, Popp A, et al. Different response to eccentric and concentric training in older men and women. Eur J Appl Physiol. 2009; 107: 145-153.

17. Watanabe $Y$, Madarame $H$, Ogasawara R, Nakazato $K$, Ishii N. Effect of very low-intensity resistance training with slow movement on muscle size and strength in healthy older adults. Clin Physiol Funct Imaging. 2014; 34: 463-470.

18. Scanlon TC, Fragala MS, Stout JR, Emerson NS, Beyer KS, Oliveira LP, et al. Muscle architecture and strength: Adaptations to short-term resistance training in older adults. Muscle Nerve. 2014; 49: 584-592. 
19. Barbat-Artigas S, Pinheiro Carvalho L, Rolland Y, Vellas B, Aubertin-Leheudre M. Muscle strength and body weight mediate the relationship between physical activity and usual gait speed. J Am Med Dir Assoc. 2016; 17: 1031-1036.

20. Bendall MJ, Bassey EJ, Pearson MB. Factors affecting walking speed of elderly people. Age Ageing. 1989; 18: 327-332.

21. Baechle TR, Westcott WL. Fitness professional's guide to strength training for older adults. 2nd ed. Champaign: Human Kinetics; 2010.

22. Kraemer WJ, Adams K, Cafarellic E, Dudley GA, Dooly C, Feigenbaum MS, et al. American College of Sports Medicine position stand: Progression models in resistance training for healthy adults. Med Sci Sports Exerc. 2002; 34: 364-380.

23. Vagetti GC, Barbosa Filho VC, Moreira NB, de Oliveira V, Mazzardo O, de Campos W. Association between physical activity and quality of life in the elderly: A systematic review, 2000-2012. Braz J Psychiatry. 2014; 36: 76-88.

24. Fragala MS, Fukuda DH, Stout JR, Townsend JR, Emerson NS, Boone $\mathrm{CH}$, et al. Muscle quality index improves with resistance exercise training in older adults. Exp Gerontol. 2014; 53: 1-6.

25. Ades PA, Ballor DL, Ashikaga T, Utton JL, Nair KS. Weight training improves walking endurance in healthy elderly persons. Ann Intern Med. 1996; 124: 568-572.

26. Westcott WL. Resistance training is medicine: Effects of strength training on health. Curr Sports Med Rep. 2012; 11: 209-216.

27. Sale DG. Neural adaptation to strength training. Strength and Power in Sport. 2nd ed. Oxford: Blackwell; 2003. p.281-p.314.

28. Morton SK, Whitehead JR, Brinkert RH, Caine DJ. Resistance training vs. static stretching: Effects on flexibility and strength. J Strength Cond Res. 2011; 25: 3391-3398.

29. Hughes KJ, Salmon N, Galvin R, Casey B, Clifford AM. Interventions to improve adherence to exercise therapy for falls prevention in community-dwelling older adults: Systematic review and meta-analysis. Age Ageing. 2019; 48: 185-195.

30. Pérez-Ros $P$, Vila-Candel R, Martínez-Arnau FM. A home-based exercise program focused on proprioception to reduce falls in frail and pre-frail community-dwelling older adults. Geriatr Nurs. 2020; 41: 436-444.

31. DiBrezzo R, Shadden BB, Raybon, BH, Powers M. Exercise intervention designed to improve strength and dynamic balance among community-dwelling older adults. J Aging Phys Act. 2005; 13: 198-209.

32. Kitagaki K, Murata S, Tsuboi Y, Isa T, Ono R. Relationship between exercise capacity and depressive symptoms in community-dwelling older adults. Arch Gerontol Geriatr. 2020; 89: 104084. 


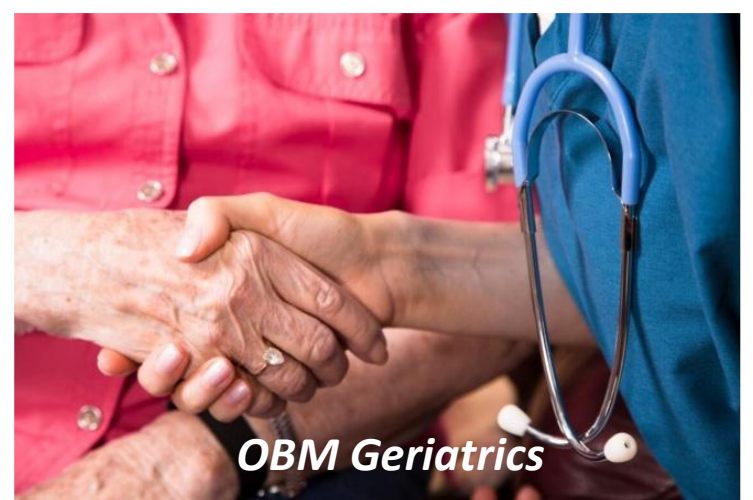

Enjoy $O B M$ Geriatrics by:

1. Submitting a manuscript

2. Joining in volunteer reviewer bank

3. Joining Editorial Board

4. Guest editing a special issue

For more details, please visit: http://www.lidsen.com/journals/geriatrics 